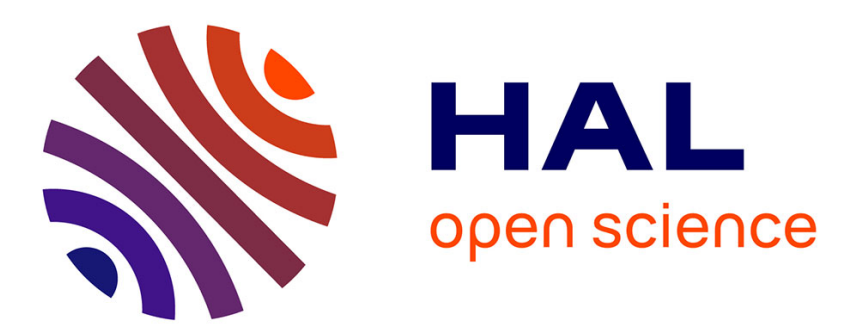

\title{
A New Reliable Numerical Method for Computing Chaotic Solutions of Dynamical Systems: The Chen Attractor Case
}

René Lozi, Alexander N. Pchelintsev

\section{- To cite this version:}

René Lozi, Alexander N. Pchelintsev. A New Reliable Numerical Method for Computing Chaotic Solutions of Dynamical Systems: The Chen Attractor Case. International journal of bifurcation and chaos in applied sciences and engineering , 2015, 25 (13), pp.1550187. 10.1142/S0218127415501874. hal-01323625

\section{HAL Id: hal-01323625 \\ https://hal.science/hal-01323625}

Submitted on 1 Jun 2016

HAL is a multi-disciplinary open access archive for the deposit and dissemination of scientific research documents, whether they are published or not. The documents may come from teaching and research institutions in France or abroad, or from public or private research centers.
L'archive ouverte pluridisciplinaire HAL, est destinée au dépôt et à la diffusion de documents scientifiques de niveau recherche, publiés ou non, émanant des établissements d'enseignement et de recherche français ou étrangers, des laboratoires publics ou privés. 


\title{
Published in \\ International Journal of Bifurcation and Chaos \\ Vol. 25, No. 13 (2015) pp. 1550187-1 to $1550187-10$ \\ DOI: $10.1142 / \mathrm{S} 0218127415501874$
}

personal file

\section{A New Reliable Numerical Method for Computing Chaotic Solutions of Dynamical Systems: The Chen Attractor Case}

\author{
René Lozi \\ University of Nice-Sophia Antipolis, Nice, \\ U.C.A., UMR CNRS 7351, France \\ rlozi@unice.fr \\ Alexander N. Pchelintsev \\ Tambov State Technical University, ul. Sovetskaya 106, \\ Tambov 392000, Russia \\ pchelintsev.an@yandex.ru
}

Received July 5, 2015

\begin{abstract}
This paper describes a new reliable numerical method for computing chaotic solutions of dynamical systems and, in special cases, is applied to Chen strange attractor. The numerical precision of the computation is finely mastered. We introduce a modification of the method of power series for the construction of approximate solutions of the Chen system together with forward/backward control of the precision. As a test for the method, we obtained the region of convergence of series and researched the behavior of the trajectories on this attractor. The results of a numerical experiment are presented.
\end{abstract}

Keywords: Chen attractor; power series; region of convergence; recurrent trajectory; Poisson stability.

\section{Introduction}

Can we trust numerical computations of chaotic solutions of dynamical systems? This question has been nagging mathematicians since the first discovery of the Lorenz strange attractor [Lozi, 2013]. Theoretical proof of the existence of such strange or hyperbolic attractor is very difficult to handle and numerical computations are in many cases not reliable due to the combined effects of rounding error and sensitive dependence on initial condition (the butterfly effect). Computer aided proofs are also complex and require special interval arithmetic analysis [Tucker, 1999]. Even nowadays, 40 years after the discovery of the first strange attractor for a mapping of the plane into itself [Henon, 1976], it is unclear if this mapping possesses a strange attractor for every value of the parameter in a small neighborhood of the classical parameter value [Galias \& Tucker, 2015].

Concerning strange attractors of ordinary differential equations, the problem seems even more difficult to deal with, requiring tremendous effort done only in the Lorenz case [Tucker, 1999].

Among such well known attractors, the Chen attractor receives considerable attention since its discovery in 1999 by Chen [Chen \& Ueta, 1999; Ueta \& Chen, 2000]. In this article, we describe a new reliable numerical method for computing its chaotic solutions. This method combines several numerical "ingredients": power series, computation of accurately jointed parts of trajectory, forward and backward computation for matching 
the required precision. It can be applied to some comparable attractors.

Let us consider the Chen system of a differential equation:

$$
\begin{aligned}
& \dot{x}=a(y-x), \\
& \dot{y}=(c-a) x-x z+c y, \\
& \dot{z}=x y-b z,
\end{aligned}
$$

where $a, b$, and $c$ are some numbers, called parameters of the system. The system (1) is typically analyzed with $a=35, b=3$, and $c=28$ (the classical values of the parameters).

The divergence of the vector field

$$
\begin{aligned}
& f(x, y, z) \\
& \quad=\{a(y-x),(c-a) x-x z+c y, x y-b z\}
\end{aligned}
$$

is

$$
\operatorname{div} f=-a-b+c<0 .
$$

This means that the system (1) is dissipative because the volume of the limit set is zero. That is, there is the compact set $M_{C} \subset \mathbb{R}^{3}$ in which all trajectories of the system (1) belong when time $t \rightarrow \infty$. Thus, all trajectories of the dynamic system (1) are attracted to the limit set, see [Nemytskii \& Stepanov, 1989, pp. 338-340]. Hence, the attractor determines the behavior of the solutions of system (1) when time goes to infinity.

In paper [Augustova \& Beran, 2013] the authors estimated the shape of $M_{C}$. Let us surround the set $M_{C}$ by the closed ball $S_{r}$ of radius $r$.

Because of the lack of accurate methods for solving nonlinear systems of ordinary differential equations of general form, many researchers use numerical methods to analyze the structure of the attractors. Some of the commonly used are a combination of the explicit Euler scheme with the central-difference scheme [Lorenz, 1963], the Adams scheme [Yorke \& Yorke, 1979], higher derivatives scheme [Sparrow, 1982], and fourth-order RungeKutta method [Kaloshin, 2001]. For the classical values of the system parameters, the solutions are unstable, since the equilibrium points of the system are the saddle type. Therefore, the above methods cannot be used, since the total error increases with increasing integration interval [Babuska et al., 1966, Sec. 3.2.3] (it cannot exceed $2 r+\delta$, where $\delta$ is rather small). Most importantly, the result cannot be improved by decreasing the integration step $\Delta t$, since the integration error has an extremum as a function of $\Delta t$. This problem can be solved by using high-accuracy calculations [MPFR Library, 2015]. However, this approach restricts the study: first, the way to decrease the error is narrow (to change $\Delta t$ and the accuracy of real number representation in order to control the calculation process). Second, the number of operations needed for very small $\Delta t$ is large. A new way to solve this problem is using the method of power series tailored in particular manner, as it is applied to the Lorenz system in [Pchelintsev, 2014].

The aim of this paper is to apply this new and very powerful method to the Chen attractor. In this scope, it is necessary first to estimate the region of convergence of the power series.

\section{Description of the Numerical Method}

To find approximate solutions of systems of differential equations, the method of power series (or the method of Taylor series) is sometimes used. The integration error of the solution can be changed by varying the accuracy of estimating the remainder term. Nowadays this method is rarely used (for second-order linear systems [Agarwal \& O'Regan, 2009]), because the calculations in the nonlinear case are cumbersome (it requires symbolic calculations). However, in some cases [Gibbons, 1960; Hairer et al., 1993; Butcher, 2003; Pchelintsev, 2014], one can get closed formulas for calculating the coefficients of expansion.

The Chen system similarly to the Lorenz one, has a polynomial right-hand side. This makes it possible to use explicit formulas to calculate the power series coefficients and estimate the region of convergence.

Let

$$
\begin{gathered}
x(t)=\sum_{i=0}^{\infty} \alpha_{i} t^{i}, \quad y(t)=\sum_{i=0}^{\infty} \beta_{i} t^{i}, \\
z(t)=\sum_{i=0}^{\infty} \gamma_{i} t^{i},
\end{gathered}
$$

where $x(0)=\alpha_{0}, y(0)=\beta_{0}$, and $z(0)=\gamma_{0}$ are initial conditions for system (1). Differentiating (2) we have

$$
\dot{x}=\sum_{i=0}^{\infty}(i+1) \alpha_{i+1} t^{i}
$$




$$
\begin{aligned}
& \dot{y}=\sum_{i=0}^{\infty}(i+1) \beta_{i+1} t^{i}, \\
& \dot{z}=\sum_{i=0}^{\infty}(i+1) \gamma_{i+1} t^{i} .
\end{aligned}
$$

The multiplications of the power series in the Cauchy form are

$$
x z=\sum_{i=0}^{\infty} \sum_{j=0}^{i} \alpha_{j} \gamma_{i-j} t^{i}, \quad x y=\sum_{i=0}^{\infty} \sum_{j=0}^{i} \alpha_{j} \beta_{i-j} t^{i} .
$$

Equating the coefficients at the same powers, we obtain from system (1) the following recurrence relations to calculate the power series coefficients in $(2)$ :

$$
\begin{aligned}
\alpha_{i+1}= & \frac{a\left(\beta_{i}-\alpha_{i}\right)}{i+1}, \\
\beta_{i+1}= & \frac{(c-a) \alpha_{i}-\sum_{j=0}^{i} \alpha_{j} \gamma_{i-j}+c \beta_{i}}{i+1}, \\
\gamma_{i+1}= & \frac{\sum_{j=0}^{i} \alpha_{j} \beta_{i-j}-b \gamma_{i}}{i+1} .
\end{aligned}
$$

Although any trajectory of system (1) is in the sphere $S_{r}$, and the right-hand side of this system is analytical everywhere, a preliminary numerical experiment demonstrated that the radius of convergence of series (2) is bounded and dependent on the initial conditions. Therefore, only a part of the trajectory can be obtained by the above method. The procedure we use consists in the construction of areas of trajectory in any time interval where series (2) converge and match those trajectory parts; which give the global desired solution. The integration error accumulated when passing from one trajectory part to another (due to an inevitable error of approximate parts of solutions) can be controlled by varying the accuracy of power series expansion. Here highly accurate calculation is necessary for finding the solution for very large time intervals, because the accuracy $\varepsilon_{p}$ of power series expansion cannot be less than the machine epsilon $\varepsilon_{m}$.

Let us consider in more detail the procedure of the construction of the arcs of trajectory in time interval $\Omega=[0 ; T]$, where the value $T$ is given.
Let $t_{l} \in \Omega, l=\overline{1, N}$ be a number of the time interval $\left[t_{l-1} ; t_{l}\right]$, where series $(2)$ converges, $N$ is the number of such intervals, $t_{0}=0, t_{N}=T$,

$$
\Omega=\left[t_{0} ; t_{1}\right] \cup\left[t_{1} ; t_{2}\right] \cup \cdots \cup\left[t_{N-1} ; t_{N}\right] .
$$

We define the values $\alpha_{0}, \beta_{0}$, and $\gamma_{0}$ of an initial condition for time $t_{0}$. Then the coefficients $\alpha_{i}, \beta_{i}$, and $\gamma_{i}(i=1,2, \ldots)$ are calculated using formulas $(3)$ until the following estimate is valid

$$
\sqrt{\alpha_{i}^{2}+\beta_{i}^{2}+\gamma_{i}^{2}}\left|\Delta t_{l}\right|^{i}<\varepsilon_{p}
$$

where $\Delta t_{l}=t_{l}-t_{l-1}$. The module in (4) is provided for negative values of the step $\Delta t_{l}$ (let us describe this later). Let $x_{1}(t), y_{1}(t)$, and $z_{1}(t)$ be the $n_{1}$-power polynomials, which are obtained for estimation (4) at a first stage $(l=1)$ of calculation. In a second stage $(l=2)$ we set

$$
\begin{gathered}
\alpha_{0}:=x_{l-1}\left(\Delta t_{l-1}\right), \quad \beta_{0}:=y_{l-1}\left(\Delta t_{l-1}\right), \\
\gamma_{0}:=z_{l-1}\left(\Delta t_{l-1}\right)
\end{gathered}
$$

and translate the initial time $t_{1}$ to zero for simplifying the calculations (since system (1) is dynamic). The $n_{l}$-power polynomials $x_{l}(t), y_{l}(t)$, and $z_{l}(t)$ are good approximations of the corresponding part of the arc of trajectory.

Let

$$
\begin{aligned}
n_{\min } & =\min _{l} n_{l}, \quad n_{\max }=\max _{l} n_{l}, \\
l_{\min } & =\underset{l}{\operatorname{indmin}} n_{l}, \quad l_{\max }=\operatorname{indmax}_{l} n_{l}, \\
\Delta t_{\min } & =\min _{l} \Delta t_{l}, \quad \Delta t_{\max }=\max _{l} \Delta t_{l}, \\
d_{\min } & =\operatorname{indmin}_{l} \Delta t_{l}, \quad d_{\max }=\operatorname{indmax}_{l} \Delta t_{l} .
\end{aligned}
$$

If $\tau_{l}$ is the region (radius) of convergence of the power series (2), then the value $\Delta t_{l}$ verifies

$$
0<\Delta t_{l}<\tau_{l},
$$

or

$$
-\tau_{l}<\Delta t_{l}<0 .
$$

The algorithm of constructing the arc of trajectory is shown in Fig. 1. The variable way allows to go back in time when its value is -1 (for going forward way $=1$ ). For the sake of simplicity, the algorithm uses the positive values of time in both directions of movement along a trajectory. Thus, the algorithm makes it possible to construct an approximate solution of forward and backward time. 


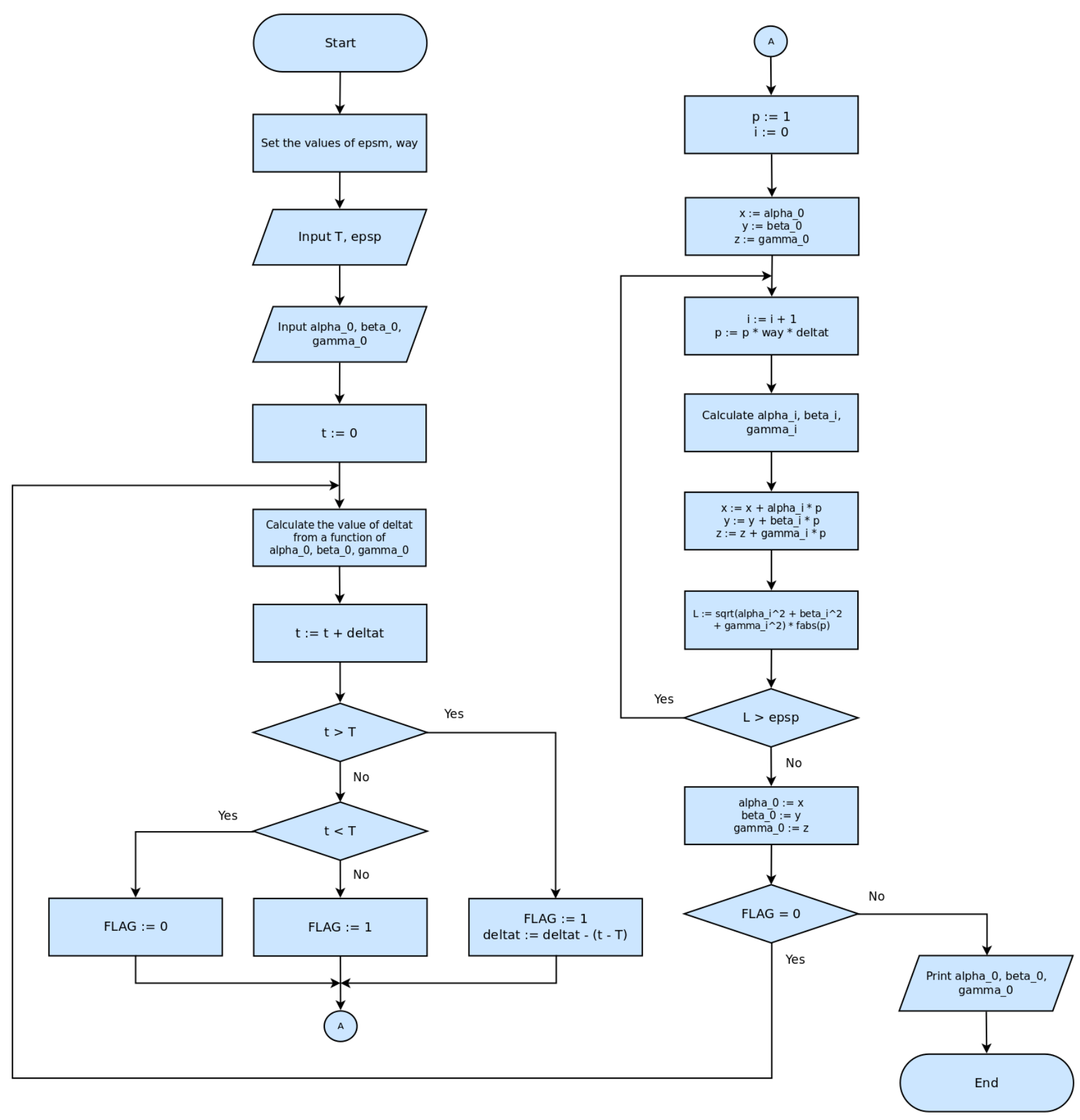

Fig. 1. The algorithm of constructing the arc of trajectory. 
We show below that the value $\Delta t_{l}$ is different for any $l$ because $\tau_{l}$ depends on $\alpha_{0}, \beta_{0}$, and $\gamma_{0}$.

\section{Estimating the Region of Convergence of the Power Series}

Estimating the region of convergence of series (2) is important when approximate solutions of system (1) are obtained by the method described above. To do this, we introduce the following notation:

$$
\begin{aligned}
& h_{1}=\max \left\{2|a|,|c-a|+|c|+2 h_{2},|b|+2 h_{2}\right\}, \\
& h_{2}=\max \left\{\left|\alpha_{0}\right|,\left|\beta_{0}\right|,\left|\gamma_{0}\right|\right\} .
\end{aligned}
$$

If $h_{2} \geq 1, h_{3}=h_{1} h_{2}$. Otherwise, $h_{3}=\max \{2|a|$, $|c-a|+|c|+1,|b|+1\}$.

Let us prove that series (2) converge for $t \in$ $\left(-\tau_{l} ; \tau_{l}\right)$, where $\tau_{l}=1 / h_{3}$. For this, the number $h_{3}$ (with $h_{3}|t|<1$ ) should be chosen such that

$$
\begin{gathered}
\left|\alpha_{i} t^{i}\right| \leq\left(h_{3}|t|\right)^{i}, \quad\left|\beta_{i} t^{i}\right| \leq\left(h_{3}|t|\right)^{i}, \\
\left|\gamma_{i} t^{i}\right| \leq\left(h_{3}|t|\right)^{i} .
\end{gathered}
$$

Then series (2) converge absolutely by the direct comparison test.

Theorem 1. The following inequalities hold

$$
\left|\alpha_{i}\right| \leq h_{3}^{i}, \quad\left|\beta_{i}\right| \leq h_{3}^{i}, \quad\left|\gamma_{i}\right| \leq h_{3}^{i}
$$

for any natural number $i$.

Proof. We use mathematical induction.

Let us consider a case where $h_{2} \geq 1$. Let us show that (5) is valid for $i=1$. From formulas (3), we have

$$
\begin{aligned}
& \alpha_{1}=a\left(\beta_{0}-\alpha_{0}\right), \\
& \beta_{1}=(c-a) \alpha_{0}-\alpha_{0} \gamma_{0}+c \beta_{0}, \\
& \gamma_{1}=\alpha_{0} \beta_{0}-b \gamma_{0} .
\end{aligned}
$$

Then

$$
\begin{aligned}
\left|\alpha_{1}\right| & \leq|a|\left(\left|\beta_{0}\right|+\left|\alpha_{0}\right|\right) \\
& \leq|a| \cdot 2 h_{2}=2|a| h_{2} \leq h_{1} h_{2}=h_{3}^{1}, \\
\left|\beta_{1}\right| & \leq|c-a|\left|\alpha_{0}\right|+\left|\alpha_{0}\right|\left|\gamma_{0}\right|+|c|\left|\beta_{0}\right| \\
& \leq|c-a| h_{2}+h_{2}^{2}+|c| h_{2} \\
& =\left(|c-a|+|c|+h_{2}\right) h_{2} \\
& \leq\left(|c-a|+|c|+2 h_{2}\right) h_{2} \leq h_{1} h_{2}=h_{3}^{1},
\end{aligned}
$$

$$
\begin{aligned}
\left|\gamma_{1}\right| & \leq\left|\alpha_{0}\right|\left|\beta_{0}\right|+|b|\left|\gamma_{0}\right| \\
& \leq h_{2}^{2}+|b| h_{2}=\left(|b|+h_{2}\right) h_{2} \\
& \leq\left(|b|+2 h_{2}\right) h_{2} \leq h_{3}^{1},
\end{aligned}
$$

which proves that (5) is valid when $i=1$.

Assume that (5) is valid for $i=k$. Then it is also valid for any $j=\overline{1, k}$, that is,

$$
\left|\alpha_{j}\right| \leq h_{3}^{j}, \quad\left|\beta_{j}\right| \leq h_{3}^{j}, \quad\left|\gamma_{j}\right| \leq h_{3}^{j} .
$$

Let us prove that (5) is valid for $i=k+1$. From formulas (3) and inequalities (6) we obtain the estimate (considering that $k \geq 1$ and $h_{2} \geq 1$ )

$$
\begin{aligned}
\left|\alpha_{k+1}\right| & \leq \frac{|a|}{k+1}\left(\left|\beta_{k}\right|+\left|\alpha_{k}\right|\right) \leq|a|\left(h_{3}^{k}+h_{3}^{k}\right) \\
& =2|a| h_{3}^{k} \leq h_{1} h_{3}^{k} \leq h_{1} h_{2} h_{3}^{k}=h_{3}^{k+1} .
\end{aligned}
$$

Given the inequality (6) we have

$$
\begin{aligned}
\left|\sum_{j=0}^{k} \alpha_{j} \gamma_{k-j}\right| & =\left|\alpha_{0} \gamma_{k}+\alpha_{k} \gamma_{0}+\sum_{j=1}^{k-1} \alpha_{j} \gamma_{k-j}\right| \\
& \leq\left|\alpha_{0}\right|\left|\gamma_{k}\right|+\left|\alpha_{k}\right|\left|\gamma_{0}\right|+\sum_{j=1}^{k-1}\left|\alpha_{j}\right|\left|\gamma_{k-j}\right| \\
& \leq h_{2} h_{3}^{k}+h_{3}^{k} h_{2}+\sum_{j=1}^{k-1} h_{3}^{j} h_{3}^{k-j} \\
& =2 h_{2} h_{3}^{k}+(k-1) h_{3}^{k},
\end{aligned}
$$$$
\left|\sum_{j=0}^{k} \alpha_{j} \beta_{k-j}\right| \leq 2 h_{2} h_{3}^{k}+(k-1) h_{3}^{k} .
$$

We have (considering that $k \geq 1$ and $h_{2} \geq 1$ )

$$
\begin{aligned}
\left|\beta_{k+1}\right| \leq & \frac{1}{k+1} \\
& \times\left(|c-a|\left|\alpha_{k}\right|+\left|\sum_{j=0}^{k} \alpha_{j} \gamma_{k-j}\right|+|c|\left|\beta_{k}\right|\right) \\
\leq & \frac{|c-a| h_{3}^{k}+2 h_{2} h_{3}^{k}+(k-1) h_{3}^{k}+|c| h_{3}^{k}}{k+1} \\
= & \frac{|c-a| h_{3}^{k}+\left(2 h_{2}-1\right) h_{3}^{k}+|c| h_{3}^{k}}{k+1} \\
& +\frac{k}{k+1} h_{3}^{k}
\end{aligned}
$$




$$
\begin{aligned}
& \leq\left(|c-a| h_{3}^{k}+2 h_{2} h_{3}^{k}-h_{3}^{k}+|c| h_{3}^{k}\right)+h_{3}^{k} \\
& =\left(|c-a|+|c|+2 h_{2}\right) h_{3}^{k} \leq h_{1} h_{3}^{k} \leq h_{3}^{k+1}, \\
\left|\gamma_{k+1}\right| & \leq \frac{1}{k+1}\left(\left|\sum_{j=0}^{k} \alpha_{j} \beta_{k-j}\right|+|b|\left|\gamma_{k}\right|\right) \\
& \leq \frac{2 h_{2} h_{3}^{k}+(k-1) h_{3}^{k}+|b| h_{3}^{k}}{k+1} \\
& =\frac{\left(2 h_{2}-1\right) h_{3}^{k}+|b| h_{3}^{k}}{k+1}+\frac{k}{k+1} h_{3}^{k} \\
& \leq\left(2 h_{2} h_{3}^{k}-h_{3}^{k}+|b| h_{3}^{k}\right)+h_{3}^{k} \\
& =\left(|b|+2 h_{2}\right) h_{3}^{k} \leq h_{1} h_{3}^{k} \leq h_{3}^{k+1},
\end{aligned}
$$

which proves that (5) is valid for any natural $i$ when $h_{2} \geq 1$.

Now, let us consider the other case $h_{2}<1$, and prove by induction that (5) is valid in this case. For $i=1$ we have

$$
\begin{aligned}
& \left|\alpha_{1}\right| \leq 2|a| \leq h_{3}^{1}, \\
& \left|\beta_{1}\right| \leq|c-a| \cdot 1+1 \cdot 1+|c| \cdot 1 \leq h_{3}^{1}, \\
& \left|\gamma_{1}\right| \leq 1 \cdot 1+|b| \cdot 1 \leq h_{3}^{1} .
\end{aligned}
$$

Hence, when $i=1$, statement (5) is valid.

Assume that (5) is valid for $i=k$.

Let us prove that (5) is valid for $i=k+1$. It follows from formulas (3) and the above assumption that

$$
\begin{aligned}
\left|\alpha_{k+1}\right| & \leq 2|a| h_{3}^{k} \leq h_{3} h_{3}^{k}=h_{3}^{k+1}, \\
\left|\sum_{j=0}^{k} \alpha_{j} \gamma_{k-j}\right| & \leq 1 \cdot\left|\gamma_{k}\right|+\left|\alpha_{k}\right| \cdot 1+(k-1) h_{3}^{k} \\
& \leq h_{3}^{k}+h_{3}^{k}+(k-1) h_{3}^{k} \\
& =(k+1) h_{3}^{k}, \\
\left|\sum_{j=0}^{k} \alpha_{j} \beta_{k-j}\right| & \leq(k+1) h_{3}^{k}, \\
\left|\beta_{k+1}\right| & \leq \frac{|c-a| h_{3}^{k}+(k+1) h_{3}^{k}+|c| h_{3}^{k}}{k+1} \\
& =\frac{|c-a| h_{3}^{k}+|c| h_{3}^{k}}{k+1}+h_{3}^{k} \\
& \leq|c-a| h_{3}^{k}+|c| h_{3}^{k}+h_{3}^{k}
\end{aligned}
$$

$$
\begin{aligned}
& =(|c-a|+|c|+1) h_{3}^{k} \\
& \leq h_{3} h_{3}^{k}=h_{3}^{k+1}, \\
\left|\gamma_{k+1}\right| & \leq \frac{(k+1) h_{3}^{k}+|b| h_{3}^{k}}{k+1} \\
& =h_{3}^{k}+\frac{|b| h_{3}^{k}}{k+1} \\
& \leq h_{3}^{k}+|b| h_{3}^{k}=(|b|+1) h_{3}^{k} \\
& \leq h_{3} h_{3}^{k}=h_{3}^{k+1},
\end{aligned}
$$

which proves that (5) is valid for any natural $i$ when $h_{2}<1$.

It should be noted that the above scheme for obtaining the convergence domain of the power series can be, by analogy, extended to other third-order dynamic systems with nonlinearities of form (1). The paper [Pchelintsev, 2014] is concerned with the Lorenz system.

\section{Numerical Results}

In this section, we present the results of computation based on the above scheme. In this example, the precision floating-point calculations are implemented on the basis GNU MPFR Library [MPFR Library, 2015], and to be more precise, the highperformance $\mathrm{C}++$ interface [Holoborodko, 2015] for MPFR library is used with arbitrary-precision real numbers in $\mathrm{C}++$. It is convenient because it has the class mpreal with the overloaded arithmetic operations and friendly mathematical functions (for instance, fabs() and sqrt()). All figures are made in the program gnuplot [Gnuplot, 2015].

In paper [Tucker, 2002, pp. 90, 91], the Euler method with a variable integration step $\Delta t$ is used to study the behavior of trajectories on the attractor. The value of $\Delta t$ taken in the calculations is chosen by error tracing at the current step (that is, by local control) using interval arithmetic. However, the total integration error is not verified. In order to remove this drawback, we use the same method time reversely from the final point to a neighborhood of the initial one of the arc of trajectory. This method guarantees that the approximate solution is constructed correctly. The error with respect to the step can be decreased by varying the accuracy $\varepsilon_{p}$, which is not allowed in the Euler method. In the above-considered modification of the power series method, an advantage over the general scheme of 


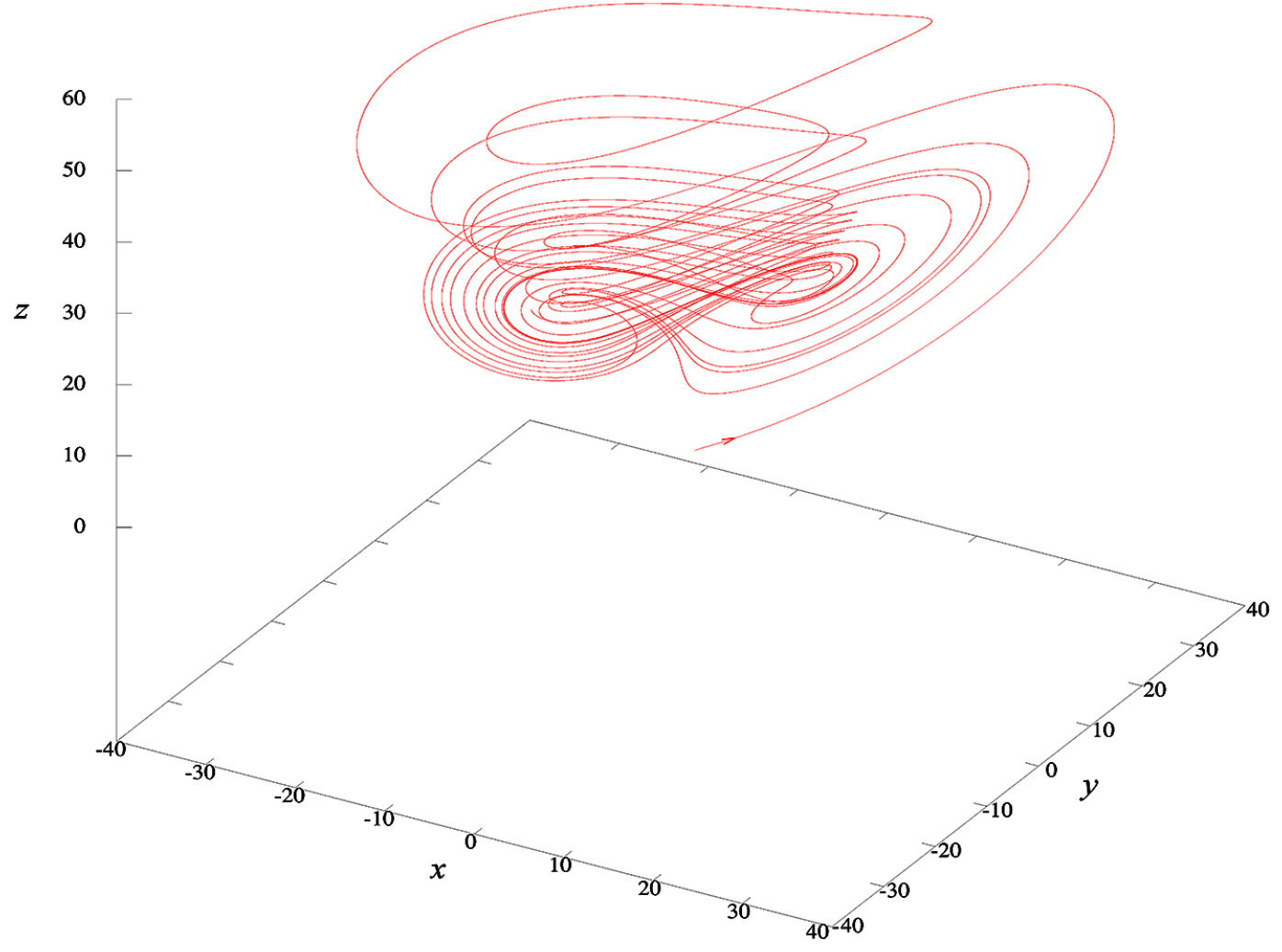

Fig. 2. The arc of trajectory constructed in the time interval $[0 ; 15]$ for $x(0)=y(0)=z(0)=1$.

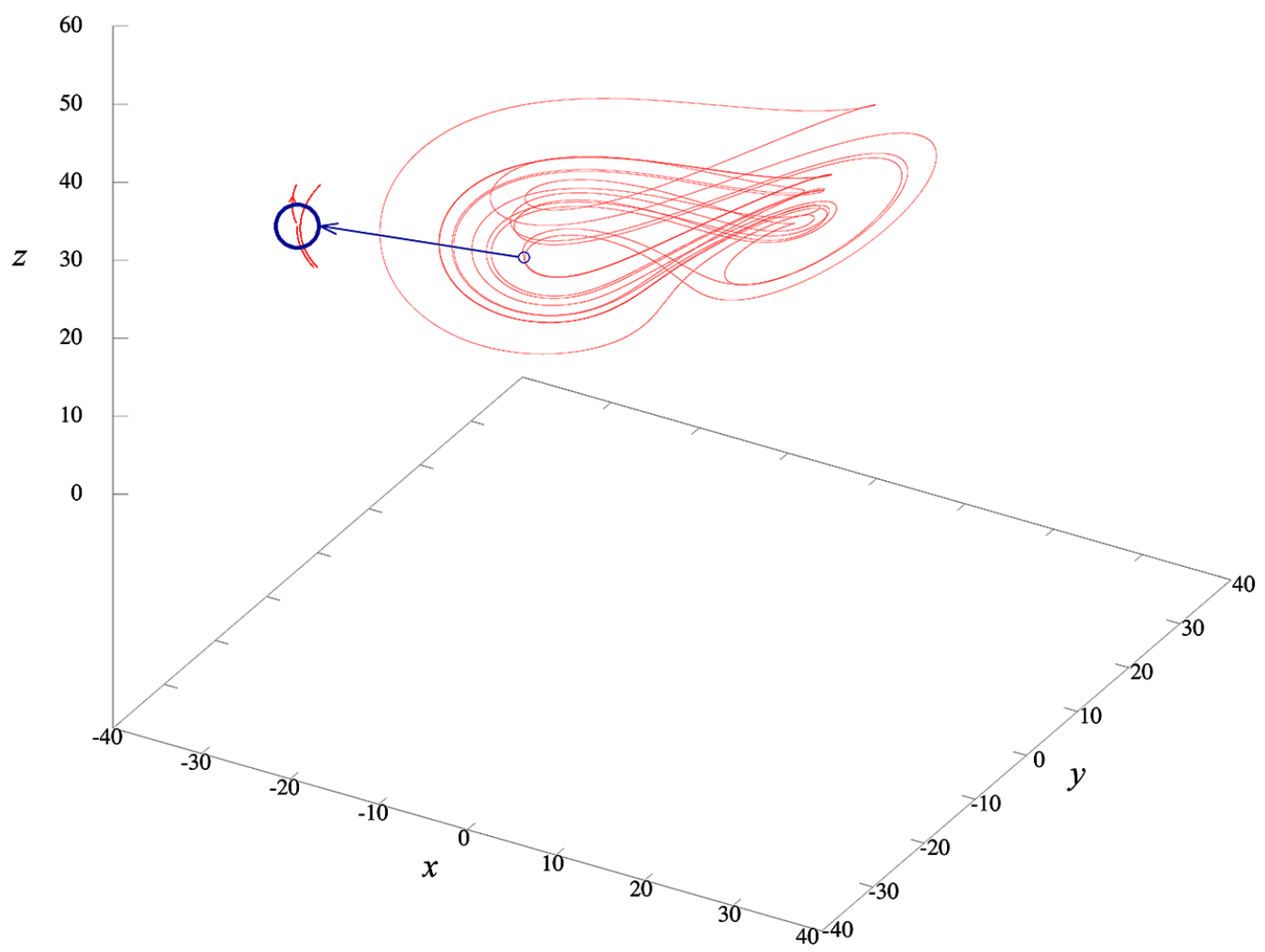

Fig. 3. The arc of trajectory constructed in the time interval $[0 ; 8.411]$ for $x(0)=-10.33913519761, y(0)=-11.10031188035$ and $z(0)=23.84877914089$. 
the Taylor series method is that the expansion coefficients can be rapidly calculated by formulas (3) in comparison to the procedure of symbol differentiation of the right-hand sides of the system equations (in the nonlinear case, much memory is needed to store the symbol expressions in the calculation of the higher-order derivatives).

To research the trajectories of the attractor (for the classical values of the system parameters) we take an arbitrary starting point and construct the arc of trajectory for the large time interval $[0 ; 15]$ :

$$
x(0)=y(0)=z(0)=1,
$$

we get the result (shown to the 11th decimal place)

$$
\begin{aligned}
& x(15)=-10.33913519761, \\
& y(15)=-11.10031188035, \\
& z(15)=23.84877914089,
\end{aligned}
$$

wherein $\varepsilon_{p}=10^{-80}, \varepsilon_{m}=9.81819 \cdot 10^{-91}$ (the number of bits for the mantissa of a real number was taken as $\left.b_{m}=300\right)$. The above-given accuracy in representing real numbers was taken to go back in time from the final point to the initial one in the trajectory. As a result, we have values coinciding with the initial ones to the third decimal place. The arc of trajectory constructed in the time interval $[0 ; 15]$ is presented in Fig. 2.

The choice of such a small value of $\varepsilon_{p}$ is due to the fact that when moving along a trajectory following reverse time, the solutions are strongly unstable: they immediately go to infinity from the attractor, since in our calculations we are close to it but not directly on it. However, we cannot always go back to the acceptable neighborhood of the start point. If a value of the accuracy $\varepsilon_{p}$ is large, then the point of the trajectory will go to infinity when going back because there is the strong unstability. Then, the value $\Delta t_{l}$ has to be very small (it follows from Sec. 3 of this paper). Based on the block diagram in Fig. 1 this fact will cause the significant deceleration of calculations.

According to Birkhoff's theorem [Nemytskii \& Stepanov, 1989, Part Two, Sec. 7], the Chen attractor contains recurrence trajectories, and every such trajectory is Poisson-stable [Nemytskii \& Stepanov, 1989, Part Two, Sec. 4]. This means that there exist arbitrarily large values of $t_{\rho}$ such that a point of the trajectory belongs to any $\rho$-neighborhood of its initial value,

$$
\eta\left(t_{\rho}\right)=\sqrt{\left(x\left(t_{\rho}\right)-x(0)\right)^{2}+\left(y\left(t_{\rho}\right)-y(0)\right)^{2}+\left(z\left(t_{\rho}\right)-z(0)\right)^{2}}<\rho .
$$

Let us use the coordinates (7) as the coordinates of the starting point because it is near to the Chen attractor. The arc of trajectory constructed in the time interval $[0 ; 8.411]$ is presented in Fig. 3. The arrow shows the trajectory when it returns to the $\rho$-neighborhood of the starting point.

We give the result in Tables 1 and 2 (not all decimal places are displayed) wherein the distance

Table 1. The values of coordinates.

\begin{tabular}{llccc}
\hline No. & \multicolumn{1}{c}{$t$} & $x(t)$ & $y(t)$ & $z(t)$ \\
\hline 1 & 0 & -10.3391 & -11.1003 & 23.8488 \\
2 & 3.695 & -10.4283 & -10.7454 & 23.3929 \\
3 & 8.411 & -10.5177 & -10.7434 & 23.5557 \\
\hline
\end{tabular}

Table 2. The values of derivatives.

\begin{tabular}{lllll}
\hline No. & \multicolumn{1}{c}{$t$} & \multicolumn{1}{c}{$\dot{x}(t)$} & \multicolumn{1}{c}{$\dot{y}(t)$} & $\dot{z}(t)$ \\
\hline 1 & 0 & -26.6412 & 8.14097 & 43.2213 \\
2 & 3.695 & -11.0986 & 16.0749 & 41.8775 \\
3 & 8.411 & -7.89935 & 20.561 & 42.3287 \\
\hline
\end{tabular}

$\eta(t)$ is equal

$$
\eta(3.695)=0.58, \quad \eta(8.411)=0.5 .
$$

To go back on time and identify all decimal places of the starting point coordinates, we must take

Table 3. The results of calculating experiment at $T=3.695$ and $T=8.411$ for $w a y=1$.

\begin{tabular}{lcc}
\hline$T$ & 3.695 & 8.411 \\
\hline$N$ & 7549 & 16869 \\
$n_{\min }$ & 18 & 19 \\
$l_{\min }$ & $N$ & $N$ \\
$n_{\max }$ & 27 & 27 \\
$l_{\max }$ & 2304 & 2304 \\
$t_{l_{\min }}$ & 3.69487 & 8.41082 \\
$t_{l_{\max }}$ & 1.26107 & 1.26107 \\
$\Delta t_{\min }$ & 0.000129384 & 0.000179493 \\
$d_{\min }$ & $l_{\min }$ & $l_{\min }$ \\
$t_{d_{\min }}$ & $t_{l_{\min }}$ & $t_{l_{\min }}$ \\
$\Delta t_{\max }$ & 0.00124324 & 0.00124324 \\
$d_{\max }$ & 2299 & 2299 \\
$t_{d_{\max }}$ & 1.25485 & 1.25485 \\
\hline
\end{tabular}


Table 4. The results of calculating experiment at $T=3.695$ and $T=8.411$ for way $=-1$.

\begin{tabular}{lcc}
\hline$-T$ & -3.695 & -8.411 \\
\hline$\hat{N}$ & 7549 & 16869 \\
$\hat{n}_{\min }$ & 18 & 19 \\
$\hat{l}_{\min }$ & $\hat{N}$ & $\hat{N}$ \\
$\hat{n}_{\max }$ & 27 & 27 \\
$\hat{l}_{\max }$ & 5206 & 14526 \\
$t_{\hat{l}_{\min }}$ & -3.69489 & -8.41083 \\
$t_{\hat{l}_{\max }}$ & -2.38689 & -7.10279 \\
$\Delta t_{\max }$ & -0.000109911 & -0.000165672 \\
$\hat{d}_{\max }$ & $\hat{l}_{\min }$ & $\hat{l}_{\min }$ \\
$t_{\hat{d}_{\max }}$ & $t_{\hat{l}_{\min }}$ & $t_{\hat{l}_{\min }}$ \\
$\Delta t_{\min }$ & -0.00124325 & -0.00124325 \\
$\hat{d}_{\min }$ & 5252 & 14572 \\
$t_{\hat{d}_{\min }}$ & -2.44001 & -7.15587 \\
\hline
\end{tabular}

$$
\begin{aligned}
& 0<\varepsilon_{p} \leq 10^{-53}, \\
& 0<\varepsilon_{m} \leq 2.54895 \cdot 10^{-57} \quad\left(b_{m} \geq 189\right) .
\end{aligned}
$$

This computation also shows the results in Tables 3 and 4 ( $t$ is counted from 0 to $-T$ for Table 4).

We then get

$$
\begin{gathered}
N=\hat{N}, \quad t_{l_{\max }}+\left|t_{\hat{l}_{\max }}\right| \approx T, \\
d_{\max }+\hat{d}_{\min } \approx N, \quad t_{d_{\max }}+\left|t_{\hat{d}_{\min }}\right| \approx T,
\end{gathered}
$$

which also confirms the correctness of the data in the calculating experiment.

\section{Conclusion}

The calculations have shown that in the Chen system the dynamical behavior of the solutions on the attractor is rather complicated (see the animation [YouTube, 2015]), and the recurrence trajectories in the attractor can, for instance, be described as almost periodic solutions or have a more complicated structure [Pchelintsev, 2013]. From an analysis of the derivatives in Table 2, we can say that the trajectory is not closed, wherein the computational error is much smaller than the radius $\rho$ neighborhood of the starting point we choose.

In the computing experiment, error accumulates at each step. As a result, we get the point either on another recurrent trajectory, or on a trajectory near the attractor, or on the same trajectory but to the point corresponding to another moment of time, due to the Poisson stability.

\section{References}

Agarwal, R. P. \& O'Regan, D. [2009] Ordinary and Partial Differential Equations (With Special Functions, Fourier Series, and Boundary Value Problems) (Springer, NY).

Augustova, P. \& Beran, Z. [2013] "Characteristics of the Chen attractor," Nostradamus 2013: Prediction, Modeling and Analysis of Complex Systems, Advances in Intelligent Systems and Computing, Vol. 210, pp. 305-312.

Babuska, I., Prager, M. \& Vitasek, E. [1966] Numerical Processes in Differential Equations (SNTL, Praha; John Wiley \& Sons, London-NY-Sydney).

Butcher, J. C. [2003] Numerical Methods for Ordinary Differential Equations (John Wiley \& Sons, Chichester).

Chen, G. \& Ueta, T. [1999] "Yet another chaotic attractor," Int. J. Bifurcation and Chaos 9, 14651466.

Galias, Z. \& Tucker, W. [2015] "Is the Henon attractor chaotic?" Chaos 25, 033102-1-12.

Gibbons, A. [1960] "A program for the automatic integration of differential equations using the method of Taylor series," Comput. J. 3, 108-111.

Gnuplot [2015] Official Website, http://www.gnuplot. info/.

Hairer, E., Norsett, S. P. \& Wanner, G. [1993] Solving Ordinary Differential Equations I: Nonstiff Problems (Springer, Berlin).

Henon, M. [1976] "Two-dimensional mapping with a strange attractor," Commun. Math. Phys. 50, 69-77.

Holoborodko, P. [2015] The High-Performance C++ Interface for MPFR Library, http://www.holoborodko.com/pavel/mpfr/.

Kaloshin, D. A. [2001] "Search for and stabilization of unstable saddle cycles in the Lorenz system," Diff. Eqs. 37, 1636-1639.

Lorenz, E. N. [1963] "Deterministic nonperiodic flow," J. Atmosph. Sci. 20, 130-141.

Lozi, R. [2013] "Can we trust in numerical computations of chaotic solutions of dynamical systems?" Topology and Dynamics of Chaos, eds. Letellier, Ch. \& Gilmore, R., World Scientific Series in Nonlinear Science Series A, Vol. 84, pp. 63-98.

MPFR Library [2015] for Multiple-Precision FloatingPoint Computations with Correct Rounding, http:// www.mpfr.org.

Nemytskii, V. V. \& Stepanov, V. V. [1989] Qualitative Theory of Differential Equations (Dover Publications, NY).

Pchelintsev, A. N. [2013] "On constructing generally periodic solutions of a complicated structure of a nonautonomous system of differential equations," Numer. Anal. Appl. 6, 54-61. 
Pchelintsev, A. N. [2014] "Numerical and physical modeling of the dynamics of the Lorenz system," Numer. Anal. Appl. 7, 159-167.

Sparrow, C. [1982] The Lorenz Equations: Bifurcations, Chaos, and Strange Attractors (Springer, NY).

Tucker, W. [1999] "The Lorenz attractor exists," Comptes-Rendus de l'Academie des Sciences (Paris), I 328, 1197-1202.

Tucker, W. [2002] "A rigorous ODE solver and Smale's 14th problem," Found. Comput. Math. 2, 53-117.
Ueta, T. \& Chen, G. [2000] "Bifurcation analysis of Chen's attractor," Int. J. Bifurcation and Chaos 10, 1917-1931.

Yorke, J. A. \& Yorke, E. D. [1979] "Metastable chaos: The transition to sustained chaotic behavior in the Lorenz model," J. Statist. Phys. 21, 263-277.

YouTube [2015] The Recurrent Trajectory on the Chen Attractor, http://www.youtube.com/watch?v= x0rndC1-qCE. 